\title{
GENERIC APPROACH FOR VISIBLE WATERMARKING
}

\author{
S. Lilly Anusha \\ Dept. of EEE, \\ S V U College of Engineering, \\ Tirupati-517502, India
}

\author{
B. AnuRadha, \\ Dept. of ECE, \\ S V U College of Engineering, \\ Tirupati-517502,India
}

\begin{abstract}
In this paper generic image watermarking technique is used for the copyright protection of color images. Watermarking with monochrome and translucent images based on One-to-One compound mapping of the values of the image pixels, which provide us the recovered image without any loss. Both the translucent full color and Opaque monochrome images are used in this paper. Two-fold monotonically increasing compound mapping is used to get more typical visible watermarks in the image. Measures have been taken to protect it from hackers.
\end{abstract}

KEYWORDS: Alpha Blending, one-to-one compound mapping, parameter randomization, mapping randomization, translucent watermark, two-fold monotonically.

\section{INTRODUCTION:}

Digital Image watermarking methods are usually classified into two types: visible and invisible [1-7]. The invisible watermarking aims to embed copyright information into host media, in case of copyright infringements, to identify the ownership of the protected host the hidden information can be retrieved. It is important that the watermarked image must be resistant to common image operations which ensure that the hidden information after alterations is still retrievable. On the other hand, methods of the visible watermarking yield visible watermarks. These visible watermarks are generally clearly visible after applying common image operations. In addition, ownership information is conveyed directly on the media and copyright violations attempts can be deterred.

In general Embedding of watermarks, degrade the quality of the host media. The legitimate users are allowed to remove the embedded watermark and original content can be restored as needed using a group of techniques, namely reversible watermarking [8-11]. However, lossless image recovery is not guaranteed by all reversible watermarking techniques, which means that the recovered image is same as the original. Lossless recovery is important where there is serious concerns about image quality such as include forensics, military applications, historical art imaging, or medical image analysis.

The most common approach is to embed a monochrome watermark using deterministic and reversible mappings of pixel values or DCT coefficients in the watermark region $[6,9,11]$. Another is to rotate consecutive watermark pixels to embed watermark that is visible [11].the watermarks of arbitrary sizes can be embedded into any host image.

Only binary visible watermarks can be embedded using these approaches.

A new method for lossless visible watermarking is proposed by using compound mappings which allow mapped values to be controllable The approach is generic, leading to the possibility of embedding different types of visible watermarks into cover images. Two applications of the proposed method are demonstrated; where we can embed opaque monochrome watermarks and nonuniformly translucent full-color ones into color images.

\section{One to One compound mapping:}

2.1Reversible one to one compound mapping:

Here, we propose a generic one-to-one compound mapping for converting a set of numerical values

$\mathrm{P}=\{\mathrm{p} 1, \mathrm{p} 2, \ldots \mathrm{pM}\}, \mathrm{Q}=\{\mathrm{q} 1, \mathrm{q} 2, \ldots \mathrm{qM}\}$, such that the mapping pi, qi for all $\mathrm{i}=1,2,3 . . \mathrm{M}$ is reversible. Here, all the values of pi and qi are image pixel values (grayscale or color values) which are investigated for copyright protection applications.. The compound mapping $\mathrm{f}$ is governed by a one-to-one function $\mathrm{F}_{\mathrm{x}}$ with one parameter $\mathrm{x}=\mathrm{a}$ or $\mathrm{b}$ in the following way. $\mathrm{q}=\mathrm{f}(\mathrm{p})=\mathrm{F}_{\mathrm{b}}^{-1}\left(\mathrm{~F}_{\mathrm{a}}(\mathrm{P})\right)$----- $(1)$

Where $F_{x}^{-1}$ the inverse of $F_{x}$, the one-to-one property leads to the fact that if $F_{a}(p)=P^{\prime}$, then $F_{a}^{-1}\left(p^{\prime}\right)=p$ for all values of $a$ and $b$. On the other hand $F_{a}(p)$, and $F_{b}(p)$ generally are set to be unequal if $\mathrm{a} \neq \mathrm{b}$.

The compound mapping described by (1) is reversible, that is $\mathrm{p}$ can be derived exactly from $\mathrm{q}$ using the following formula:

$$
\mathrm{p}=\mathrm{f}^{-1}(\mathrm{q})=\mathrm{Fa}^{-1}\left(\mathrm{~F}_{\mathrm{b}}(\mathrm{q})\right) \text {-----(2) }
$$

Lemma 1 (Reversibility of compound Mapping):

If $q=F_{b}^{-1}\left(F_{a}(p)\right)$ for any one-to-one function $F_{x}$ with $a$ parameter $x$, then $p=F_{a}^{-1}\left(F_{b}(q)\right)$ for any values of $a, b, p$ and $q$. Proof: substituting (1) into $F_{a}^{-1}\left(F_{b}(q)\right)$, We get $F_{a}^{-1}\left(F_{b}(q)\right)=F_{a}^{-1}$ $\left(\mathrm{F}_{\mathrm{b}}\left(\mathrm{F}_{\mathrm{b}}^{-1}\left(\mathrm{~F}_{\mathrm{a}}(\mathrm{P})\right)\right)\right)$.

By regarding $F_{a}(p)$ as a value $c$, the right-hand side becomes $\mathrm{F}_{\mathrm{a}}^{-1}\left(\mathrm{~F}_{\mathrm{b}}\left(\mathrm{F}_{\mathrm{b}}{ }^{-1}(\mathrm{c})\right)\right)$ which after $\mathrm{F}_{\mathrm{b}}$ and $\mathrm{F}_{\mathrm{b}}^{-1}$ are cancelled out, becomes $\mathrm{F}_{\mathrm{a}}^{-1}(\mathrm{c})$. But $\mathrm{F}_{\mathrm{a}}{ }^{-1}(\mathrm{c})=\mathrm{F}_{\mathrm{a}}{ }^{-1}\left(\mathrm{~F}_{\mathrm{a}}(\mathrm{p})\right)$ which is just $\mathrm{p}$ after $\mathrm{F}_{\mathrm{a}}$ and $\mathrm{F}_{\mathrm{a}}^{-1}$ are cancelled out. hence proved $\mathrm{p}=\mathrm{F}_{\mathrm{a}}^{-1}\left(\mathrm{~F}_{\mathrm{b}}(\mathrm{q})\right)$

As an example, if $F_{x}(p)=x p+d$, then $F_{x}^{-1}\left(p^{\prime}\right)=\left(p^{\prime}-d\right) / x$.

$$
\text { Thus } \quad \begin{aligned}
\mathrm{q} & =\mathrm{F}_{\mathrm{b}}^{-1}\left(\mathrm{~F}_{\mathrm{a}}(\mathrm{p})\right) \\
& =\mathrm{F}_{\mathrm{b}}{ }^{-1}(\mathrm{ap}+\mathrm{d}) \\
& =(\mathrm{ap}+\mathrm{d}-\mathrm{d}) / \mathrm{b}=\mathrm{ap} / \mathrm{b}
\end{aligned}
$$

And so we have

$$
\begin{aligned}
\mathrm{F}_{\mathrm{a}}^{-1}\left(\mathrm{~F}_{\mathrm{b}}(\mathrm{q})\right) & =\mathrm{F}_{\mathrm{a}}^{-1}(\mathrm{~b}(\mathrm{ap} / \mathrm{b})+\mathrm{d}) \\
& =\mathrm{F}_{\mathrm{a}}^{-1}(\mathrm{ap}+\mathrm{d}) \\
& =[((\mathrm{ap}+\mathrm{d})-\mathrm{d}) / \mathrm{a}] \\
& =a \mathrm{p} / \mathrm{a} \\
& =\mathrm{p}
\end{aligned}
$$




\subsection{Lossless Visible Watermarking}

The proposed generic lossless visible watermarking using one-to-one compound mappings will be derived using the lemma 1, using which a variety of visible watermarks can be embedded into images. The embedding is reversible; the original image is recovered losslessly by removing the water mark. A preliminary lemma is first described as follows.

Lemma 2(preference of compound-mapped value $q)$ : it is possible to use the compound mapping $\mathrm{q}=\mathrm{F}_{\mathrm{b}}{ }^{-1}\left(\mathrm{~F}_{\mathrm{a}}(\mathrm{p})\right)$ to convert a numeric value $\mathrm{p}$ to other value which is close to a preferred value

Proof: Let $F_{x}(p)=p-x$ where $x$ is the parameter for $F$. then $\mathrm{F}_{\mathrm{x}}{ }^{-1}\left(\mathrm{p}^{\prime}\right)=\mathrm{p}^{\prime}+\mathrm{x}$. Also, let $\mathrm{a}=\mathrm{p}-\varepsilon$ and $\mathrm{b}=1$ where $\varepsilon$ is a small value. Then the compound mapping $F_{b}^{-1}\left(F_{a}(p)\right)$ of $p$ yields $q$ as

$$
\begin{aligned}
\mathrm{q} & =\mathrm{f}(\mathrm{p}) \quad=\mathrm{F}_{\mathrm{b}}{ }^{-1}\left(\mathrm{~F}_{\mathrm{a}}(\mathrm{P})\right) \\
& =\mathrm{F}_{\mathrm{b}}{ }^{-1}(\mathrm{p}-\mathrm{a})=\mathrm{F}_{\mathrm{b}}{ }^{-1}(\varepsilon) \\
& =\varepsilon+\mathrm{b}=\varepsilon+1 \quad---(4)
\end{aligned}
$$

This means that the value $\mathrm{q}$ is close to the preferred value 1 .

The above lemma is based on two assumptions. The first ' $a$ ' is close to ' $p$ ', or equivalently, that $a=p-\varepsilon$. The reason why we derive the above lemma for $a=p-\varepsilon$ instead of $a=p$ is that in the reverse mapping we want to recover $\mathrm{p}$ from $\mathrm{q}$ without knowing $\mathrm{p}$, which is a requirement in the application of reversible visible watermarking investigated in this studies. Although the value of $p$ cannot be known in advance for such applications, it can usually be estimated, and some techniques are described for such estimations.

The second assumption is that Fx ( $p$ ) yield a small value if $\mathrm{x}$ and $\mathrm{p}$ are close.

\section{Theorem 1: (Lossless Reversible Visible Watermarking)}

There exist one-to-one compound mappings for use to embed into a given image a visible watermark whose pixel values are close to those of a given watermark, such that the original image can be recovered from losslessly.

Proof:

This is a consequence of Lemmas 1 and 2 after regarding the individual pixel values in I, $\mathrm{L}$ and $\mathrm{Q}$ respectively as those of $\mathrm{p}, 1$ and $q$ mentioned in Lemma 2. And it is clear by Lemma 1 that the value $\mathrm{p}$ can be recovered losslessly from the mapped value $\mathrm{q}$ which is derived in Lemma 2.

\section{Algorithm 1:}

Input: original image, watermark image.

Output: watermarked image (W).

\section{Steps:}

1) From the image I Select a set of P pixels in the res where the watermark is to be embedded, call $\mathrm{P}$ a watermarking area.

2) Corresponding set of pixels $P$ in $W$ are denoted by $Q$.

3) For each pixel $\mathrm{X}$ with value $\mathrm{p}$ in $\mathrm{P}$, corresponding pixel in $\mathrm{Q}$ is denoted as $\mathrm{Z}$ and corresponding pixel $\mathrm{Y}$ in $\mathrm{L}$ as 1 and the conduct the following steps:

a) Apply an estimation technique to derive a to be a value close to $\mathrm{b}$ using the value of the neighboring pixels of $\mathrm{X}$

b) Set ' $b$ ' to be the value ' $l$ ',

c) Map ' $p$ ' to a new value $q=F_{b}^{-1}\left(F_{a}(p)\right)$.

d) The value of $Z$ is set to $q$.

Now, Set each remaining pixel value in $\mathrm{W}$, outside the region $\mathrm{P}$, to be equal to the corresponding pixel in ' $\mathrm{I}$ '.

Here we do not use the information of the original image pixel value of $\mathrm{X}$ itself for computing the parameters $\mathrm{a}$ and $\mathrm{b}$ for $\mathrm{X}$.

As an example, the purpose performed by Step: 3 of the above algorithm for a pixel is illustrated by figure. Here the color of the center pixel is estimated by using west and north pixels As the pixels are unknown to the receiver and covered by the watermark the east and south pixels are not used. Following algorithm describes removal process for watermarked image.

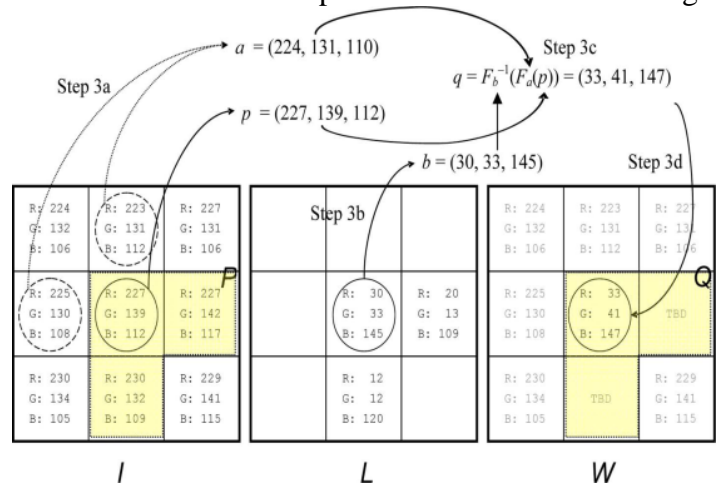

Figure 1: mapping of center pixel of a $3 \times 3$ image.

\section{Algorithm 2:}

Input: watermarked-image (W), a watermark (L). Output: recovered image $\mathrm{R}$ from $\mathrm{W}$.

Steps:

1) The watermarking area $\mathrm{Q}$ is selected in $\mathrm{W}$ as the area selected in Algorithm 1.

2) Value of each pixel in $R$ is set, which is outside the region $Q$, to be equal to the corresponding pixel in $\mathrm{W}$.

3) For each pixel $\mathrm{Z}$ with value $\mathrm{q}$ in $\mathrm{Q}$, denote the corresponding pixel in the recovered image $R$ as $X$ and the value of the corresponding pixel $\mathrm{Y}$ in $\mathrm{L}$ as 1 , and conduct the following steps.

a) Obtain the same value by applying the same estimation technique used.

b) Set $b$ to be the value 1 .

c) Restore $\mathrm{p}$ from $\mathrm{q}$ by setting $\mathrm{p}=\mathrm{F}_{\mathrm{a}}^{-1}\left(\mathrm{~F}_{\mathrm{b}}(\mathrm{q})\right.$ ).

d) Set the value of $X$ to be $p$.

\subsection{Security considerations:}

We want legitimate users to be able to recover the original image from a watermarked one; do not want an attacker to be able to do the same. Herein, we propose some security protection measures against illicit recoveries of original images. First, we make the parameters $a$ and $b$ in the above algorithms to be dependent on certain secret keys that are known only by the creator of the watermarked image and the intended receivers. This can be achieved by a simple technique that generate a pseudo-random sequence of numerical values using a secret key and these values are added to either or both of $\mathrm{a}$ and $\mathrm{b}$ pixels values in watermarking area and referred to as parameter randomization.

Another way of security protection is to make the choices of the positions for the pixels to be dependent on a secret key. Specifically, we propose to process two randomly chosen pixels (based on the security key) $\mathrm{P}$ in simultaneously as follows. Let the two pixels be denoted as $X_{1}$ and $X_{2}$ with values $\mathrm{p}_{1}$ and $\mathrm{P}_{2}$, respectively. The color estimates $\mathrm{a}_{1}$ and $\mathrm{a}_{2}$ corresponding to $\mathrm{X}_{1}$ and $\mathrm{X}_{2}$, respectively, are individually 
derived as before using their respective neighbors. The parameters $b_{1}$ and $b_{2}$ are set to be the values $l_{1}$ and $l_{2}$ of the respective watermark pixels $Y_{1}$ and $Y_{2}$. Then, instead of setting the values of the watermarked pixels $\mathrm{Z}_{1}$ and $\mathrm{Z}_{2}$ to be $\mathrm{q}_{1}=\mathrm{F}_{\mathrm{b} 1}{ }^{-1}$ $\left(\mathrm{F}_{\mathrm{a} 1}\left(\mathrm{p}_{1}\right)\right)$ and $\mathrm{q}_{2}=\mathrm{F}_{\mathrm{b} 2}{ }^{-1}\left(\mathrm{~F}_{\mathrm{a} 2}\left(\mathrm{p}_{2}\right)\right)$ as before, we swap the parameters and set $\mathrm{q}_{1}=\mathrm{F}_{\mathrm{b} 1}{ }^{-1}\left(\mathrm{~F}_{\mathrm{a} 2}\left(\mathrm{p}_{2}\right)\right)$ and $\mathrm{q}_{2}=\mathrm{F}_{\mathrm{b} 2}{ }^{-1}\left(\mathrm{~F}_{\mathrm{a} 1}\left(\mathrm{p}_{1}\right)\right)$.

The effectiveness of lossless recoverability does not effect by this parameter exchange, because the original pixel values can be recovered by the following compound mappings: $\mathrm{P}_{1}=\mathrm{F}_{\mathrm{a} 1}^{-1}\left(\mathrm{~F}_{\mathrm{b} 2}\left(\mathrm{q}_{2}\right)\right) \& \mathrm{p}_{2}=\mathrm{F}_{\mathrm{a} 2}{ }^{-1}\left(\mathrm{~F}_{\mathrm{b} 1}\left(\mathrm{q}_{1}\right)\right)$. This technique is referred as mapping randomization..

Last, the position in the image where a watermark is embedded affects the resilience of the watermarked image against illicit image recovery attempts. In more detail, if the watermark is embedded in a smooth region of the image, an attacker can simply fill the region with the background color to remove the watermark irrespective of the watermarking technique used. The techniques such as adaptive positioning can be used to choose an appropriate position while embedding a watermark

\section{Experimental Results}

In our experimental results for One-to-one compound mapping different color images of different sizes are used as cover images. And different logo images are used.

We measure the quality of watermarked images in terms of PSNR (Peak Signal to Noise Ratio) and MSE (Mean Square Error). In ideal case PSNR should be infinite and MSE should be zero. But it is not possible for watermarked image. So, large PSNR and small MSE is desirable. To see that if the recovered watermark is identical to the one that is embedded we calculate only MSE. In this case it should be zero.

\subsection{One to one compound mapping:}

\section{i. Lossless visible watermarking of opaque} monochrome watermark:

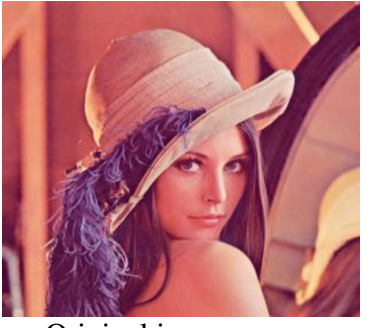

Original image

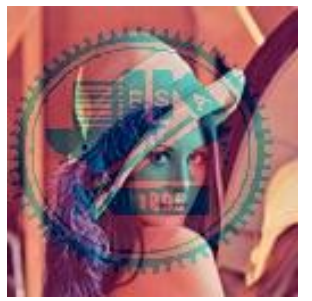

Recovered image

Figure 2: Experimental results of monochrome watermark embedding and removal.

The image has been recovered with MSE and PSNR values of

$$
\begin{aligned}
& \mathrm{MSE}=23.6935 \mathrm{~dB} \mathrm{PSNR}=34.4185 \mathrm{~dB} \\
& \text { ii. Lossless visible watermarking of }
\end{aligned}
$$
translucent color watermarks:

For ease of discussions and comparisons, we always embed a watermark in the upper left-hand corner of an image in this study.

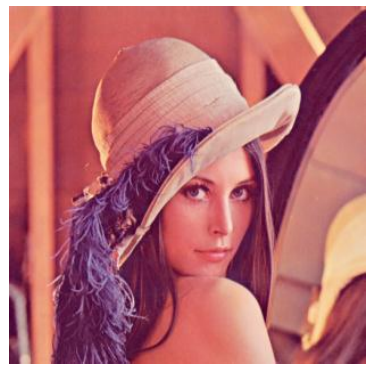

Original image

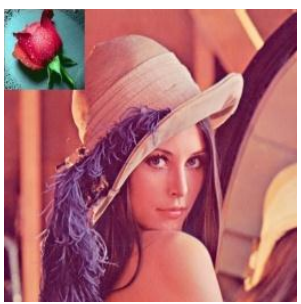

Watermarked image

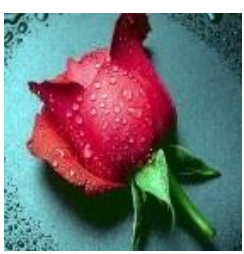

Watermark image

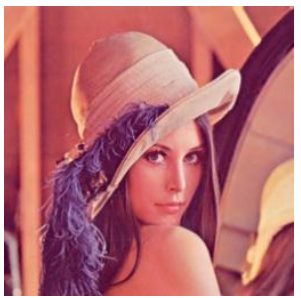

Rrecovered image
Figure 3: Experimental results of translucent watermark embedding and removal.

The image has been recovered with MSE and PSNR values of $\mathrm{MSE}=10.0308 \mathrm{~dB}$ PSNR $=38.1515 \mathrm{~dB}$

\subsection{Security Increase Results}

In our watermarking method we used encryption. So in any case if watermarking key is leaked and attacker extracts the watermark, still he will not be able to read the watermark because it is encrypted.

In our watermarking method user need two keys for watermark extraction. If any of keys is invalid then user will not be able to extract watermark correctly. The watermark will be incorrect. It depends upon which key is invalid.

\section{Conclusions:}

One to one compound mapping:

A new method with a capability of lossless image recovery for reversible visible watermarking is given. one-toone compound mappings is used which can map image pixel values to those of the desired visible watermarks. Relevant theorems and lemmas are described and proved to demonstrate the reversibility of the compound mappings for lossless reversible visible watermarking. Different types of visible watermarks are allowed to embed by compound mapping, and opaque monochrome watermarks as well as translucent fullcolor ones are described a as applications. A translucent watermark is clearly visible and visually appealing, thus more appropriate than traditional transparent binary watermarks in terms of advertising effect and copyright declaration. The two- 
fold monotonically increasing property of compound mappings was defined and an implementation proposed that can provably allow mapped values to always be close to the desired watermark if color estimates are accurate. Also described are parameter randomization and mapping randomization techniques, which can prevent illicit recoveries of original images without correct input keys. Experimental results have demonstrated the feasibility of the proposed method and the effectiveness of the proposed security protection measures.

\section{Future scope:}

Watermarking is an emerging research area for copyright protection and authentication of electronic documents and media. Most of the research is going on in this field, spatially in the field of image watermarking. The reason might be that there are so many images available at Internet without any cost, which needs to be protected.

The watermarking technique that is given in this thesis can be further improved to increase the hiding capacity of images without affecting the imperceptibility of the images.

Future research may be applied to more applications of the proposed One to one compound mapping method and extensions of the method to other data types other than bitmap images, like DCT coefficients in JPEG images and MPEG videos.

\section{REFERENCES:}

[1] G. Braudaway, K. A. Magerlein, and F. Mintzer, "Protecting publicly available images with a visible image watermark," in Proc. SPIE Int. Conf. Electronic Imaging, Feb. 1996, vol. 2659, pp. 126-133.

[2] I. J. Cox, J. Kilian, F. T. Leighton, and T. Shamoon, "Secure spread spectrum watermarking for multimedia," IEEE Trans. Image Process., vol. 6, no. 12, pp. 1673-1687, Jun. 1997.

[3] F.A.P Patitcolas, R.J.Anderson, and M.G.Kun"Information hiding- A survey"Proc.IEEE,vol:87, no.7, jul. 1999.

[4] M. S. Kankanhalli, Rajmohan, and K. R. Ramakrishnan, "Adaptive visible watermarking of images," in Proc. IEEE Int. Conf. Multimedia Computing and Systems, 1999, vol. 1, pp. 568-573.

[5] S. P. Mohanty, K. R. Ramakrishnan, and M. S Kankanhalli, "A DCT domain visible watermarking technique for images," in Proc. IEEE Int. Conf. Multimedia and Expo, Jul. 2000, vol. 2, pp. 1029 1032.

[6] N. F. Johnson, Z. Duric, and S. Jajodia, Information Hiding. Steganography and Watermarking Attacks and Countermeasures. Boston, MA: Kluwer, 2001.

[7] Y. Hu and S.Kwong,"Wavelet domain adaptive visiblewatermarking," Electron. Lett., vol. 37, no. 20, pp. 1219-1220, Sep. 2001.

[8] Y. J. Cheng and W. H. Tsai, "A new method for copyright and integrity protection for bitmap images by removable visible watermarks and irremovable invisible watermarks," presented at the Int. Computer Symp. Workshop on Cryptology and Information Security, Hualien,Taiwan, R.O.C., Dec. 2002.

[9] P. M. Huang and W. H. Tsai, "Copyright protection and authentication of grayscale images by removable visible watermarking and invisible signal embedding techniques: A new approach," presented at the Conf. Computer Vision, Graphics and Image Processing, Kinmen, Taiwan, R.O.C., Aug. 2003.

[10] Y. Hu, S. Kwong, and J. Huang, “An algorithm for removable visible watermarking," IEEE Trans. Circuits Syst. Video Technol., vol. 16, no. 1, pp. 129133, Jan.2006.

[11] S. K. Yip, O. C. Au, C. W. Ho, and H. M. Wong, "Lossless visible watermarking," in Proc. IEEE Int. Conf. Multimedia and Expo, Jul. 2006, pp. 853-856. 